\section{Las Prácticas de Extensión de Educación Experiencial como oportunidad para integrar docencia y extensión}

\section{Milagros Rafaghelli}

Docente investigadora de la Universidad

Nacional del Litoral y de la Universidad

Nacional de Entre Ríos, Argentina.

milagrosrafaghelli@gmail.com
Integración de la docencia y la extensión /

Perspectivas

RECEPCIÓN: 24/06/16

ACEPTACIÓN FINAL: 7/09/16

\section{Resumen}

El propósito de este trabajo es ofrecer un conjunto de ideas que permitan reflexionar sobre los efectos de la participación en Prácticas de Extensión de Educación Experiencial (PEEE) de la Universidad Nacional del Litoral. Las ideas deben entenderse como hipótesis de trabajo, como provocación para introducir nuevas claves de lecturas y cernir el tema que nos convoca. Muchas de ellas surgieron en espacios en los que docentes y alumnos aportaron solidariamente sus experiencias. La pretensión es revisar supuestos y referencias conceptuales e introducir otras orientaciones. Asimismo, se describen los componentes de la evaluación en las PEEE y se formulan algunos principios que deberían estar presentes en la construcción de propuestas evaluativas situadas en las prácticas.

Palabras clave

- Educación experiencial

- Aprendizaje en comunidades de práctica

- Evaluaciones situadas

- Universidad Nacional del Litoral

\section{Resumo}

O objetivo deste trabalho é fornecer um conjunto de ideias que permitam refletir sobre os efeitos da participação em Práticas de Extensão de Educação Experimental (PEEE) da Universidad Nacional del Litoral. As ideias devem ser entendidas como uma hipótese de trabalho, como uma provocação para introduzir novas chaves de leitura e apurar a questão que nos ocupa. Muitas delas surgiram em espaços onde professores e alunos contribuíram conjuntamente com as suas experiências. $\mathrm{O}$ objetivo é rever os pressupostos e referências conceituais e introduzir outras orientações. Além disso, descrevem-se os componentes da avaliação nas PEEE e se formulam alguns princípios que deveriam estar presentes na construção de propostas avaliativas situadas nas práticas.

\section{Palavras-chave}

- Educação experiencial

- Aprendizagem em comunidades de prática

- Avaliações situadas

- Universidade Nacional del Litoral
Para citación de este artículo

Rafaghelli, M. (2016). Las Prácticas de Extensión de Educación Experiencial como oportunidad para integrar docencia y extensión. En Revista $+E$ versión digital, (6), pp. 8-15. Santa Fe, Argentina: Ediciones UNL. 


\section{Prácticas de extensión: entre lo político y lo cognitivo}

Sigue siendo una tensión teórica y práctica el modo de dar lugar a la integración docencia, extensión e investigación en la formación universitaria. La preocupación constante de los profesores por la relación entre aprendizaje y experiencia muestra que el valor de la experiencia en la formación no está puesto en duda aunque se desconozca aún el mejor modo para convertirla en una opción significativa del aprendizaje.

Las Prácticas de Extensión de Educación Experiencial (PEEE) interpelan lo establecido en el currículo universitario convencional; promueven encuentros fuera del aula entre distintos actores e instituciones y diálogos con la autenticidad de las situaciones de la vida real. Problematizan cada campo de saber disciplinar, escuchan nuevas voces y abren nuevas preguntas.

La noción de aprendizaje mediante la práctica social situada en la comunidad será una dimensión clave en el análisis que iremos proponiendo. Nos acompañan las ideas de autores como Jean Lave (1991), Chaiklin y Lave (2001), Engeström (2001), Salomon (1993) y Wenger (2001), entre otros. Sería un error afirmar que ellos sostienen las mismas ideas. Los seleccionamos porque comparten un núcleo duro de conceptos y principios, y porque el corpus de sus trabajos nos ayuda a pensar el tema de esta convocatoria. Diseñar prácticas de extensión integradas al currículo universitario implica problematizar y resignificar los propósitos, los sentidos y los instrumentos de evaluación de los aprendizajes de los estudiantes. Siempre ha sido complejo en los procesos de formación articular y sostener con coherencia el deseo y su concreción. Mientras que la intención es que la evaluación sea una instancia más de aprendizaje, lo que se les solicita a los estudiantes es que respondan a preguntas que buscan verificar y controlar los saberes. Presentaremos en la segunda parte del texto algunas ideas que aporten elementos para elaborar situaciones evaluativas sensibles a las PEEE.

\section{2. ¿Cómo y qué se aprende en las Prácticas de Extensión de Educación Experiencial?}

Sin entusiasmar al lector en cuanto a que encontrará en lo que sigue una respuesta certera a los interrogantes formulados, nos interesa ensayar un marco de referencia conceptual que permita la articulación de algunas perspectivas para describir y explicar algo de lo que se pone en juego cuando la enseñanza, el aprendizaje y la evaluación se integran con la extensión.

En el campo de la Psicología de la Educación existen distintos enfoques que explican el aprendizaje, cada uno destaca aspectos diferentes del mismo. Las diferencias son de carácter fundamental en cuanto se refieren a la naturaleza del conocimiento y a los modos de conocer; al papel de la cultura y de los contenidos en el aprendizaje; al lugar de los sujetos de la educación y al de los profesionales en la sociedad. Ningún enfoque sustituye a otro, ni es en sí mismo mejor que otro, sino que cada uno de ellos toma cuerpo en supuestos distintos. Cada profesor adherirá al que mejor refleje sus intenciones pedagógicas. De todos modos, es infructuosa la toma de posición radicalizada por unos $u$ otros si no se dilucidan previamente los principios éticos y políticos que sostienen las razones de educar y las características del profesional universitario que se desea formar. Cada profesor adhiere al que mejor refleja sus intenciones.

\section{Dilemas cognitivos en la formación universitaria}

La formación universitaria se basa principalmente en el supuesto de que aprender es un proceso complejo de construcción individual de conocimientos y que requiere del mayor esfuerzo intelectual y dedicación por parte de los estudiantes. Además, se considera que es el resultado de la interacción que los alumnos realizan con los contenidos transmitidos por los profesores durante la enseñanza. Está presente la idea que la cantidad de contenidos y las actividades prácticas que sirven para su aplicación aumentan las posibilidades de comprensión. En muchas prácticas de enseñanza los estudiantes toman asiento en las aulas y/o laboratorios, prestan atención a sus profesores, anotan una gran cantidad de información y resuelven un importante número de trabajos prácticos a través de los cuales se busca promover el tan esperado proceso de construcción de conocimientos. El esfuerzo por parte de los profesores reside en seleccionar los contenidos, los ejemplos y las actividades que mejor ayuden a presentar los conceptos y las teorías que deben ser enseñados.

La Psicología Cognitiva se ha interesado por explicar cómo se produce el proceso de construcción de conocimientos y explorar la naturaleza de los cambios cognitivos. Si bien el término cognitivo se ha entendido de distintas maneras, por cognición vamos a entender aquí a los procesos, las disposiciones y la organización interna de los estudiantes que se ponen en relación entre sí y en articulación con el contenido que tiene que aprender: química analítica, sociología de las organizaciones, estrategias de comercialización, conceptos de medio ambiente y área protegida, teorías del aprendizaje, etcétera.

La actividad del aprendizaje comienza con la necesidad de entender algo nuevo. Por lo general los contenidos nuevos encuentran en los estudiantes restricciones que deben superarse, de lo contrario no habrá aprendizajes. Las reestructuraciones cognitivas globales o los cambios ocurridos en dominios específicos del saber promovidos por las explicaciones ofrecidas por los profesores son buenas estrategias para la comprensión. Si hubiese situaciones en las que algo continúa sin comprenderse, las inconsistencias se arreglan con más explicaciones o más actividades para resolver.

Los aprendizajes son el resultado de las operaciones lógicas de abstracción, reflexión y generalización que realizan los estudiantes durante la actividad intelectual. La evaluación es el combate 


\section{6}

la progresiva participación de los estudiantes en

las actividades de las comunidades de prácticas

hace que éstas se conviertan paulatinamente en

comunidades de aprendizajes

personal con el conocimiento donde debe demostrarse con certeza

y sin equívocos el resultado del proceso. La acumulación de conceptos y teorías como fin en sí mismo. La descontextualización del contenido y la actividad son condición para que se puedan aprender habilidades generales que luego serán aplicables a cualquier situación. Los estudiantes se familiarizan con un modo de aprender cuyo éxito se garantiza cuando se aprende la lógica de resolución de problemas técnicos y abstractos, aislados de cualquier distracción contextual pero con la generalidad suficiente para poder transferirlo a situaciones distintas.

Los resultados de las investigaciones en el campo de la Psicología de la Educación y la reflexión sobre intervenciones en los últimos años, comienzan a difundir la idea de que el aprendizaje de los saberes disciplinares es más potente cuando es iniciado, facilitado y consolidado en los procesos sociales y culturales reales y no artificiales. El desempeño cognitivo se enriquece cuando el aprendizaje ocurre en situaciones contextualizadas en la vida cotidiana.

Analizar el impacto de las variables situacionales en el aprendizaje no significa abandonar las propuestas de enseñanza áulicas que promueven procesos internos de construcción de conocimientos científicos.

\section{Otra idea de aprendizaje}

Permítasenos introducir otra idea de aprendizaje: como proceso y resultado de la participación social. Y otra idea de estudiante: como actor social que aprende mediante la acción en entornos culturales y resolviendo problemas de la vida reales.

Entiéndase por participación social a la interacción de manera activa de los sujetos de la educación (profesores y estudiantes) en las prácticas de una comunidad: ya sea de micro emprendedores, de ceramistas, de jóvenes ciudadanos, de vecinos de un complejo habitacional, de trabajadores de una fábrica de alimentos, etc.). La idea de aprendizaje como proceso y resultado de la participación en una comunidad de práctica, también requiere un esfuerzo intelectual, de los profesores y de los estudiantes, cargado de compromiso e interés social. La presencia de estos componentes cambia radicalmente la naturaleza y el sentido de los aprendizajes. Independientemente de cuál sea la descripción formal del perfil y las incumbencias de los profesionales que se mencionan en el currículo universitario, cada comunidad crea prácticas para hacer lo que es necesario hacer. La progresiva participación de los estudiantes en las actividades de las comunidades de prácticas hace que éstas se conviertan paulatinamente en comunidades de aprendizajes.

La actividad, o sea, eso que las personas hacen en su trabajo cotidiano, tiene una estructuración situacional específica, real y no artificial, en la que estudiantes y profesores ponen a dialogar los saberes disciplinares. La tensión entre los contenidos disciplinares y la estructuración situacional específica de las actividades en las comunidades de práctica es el núcleo principal de la idea de aprendizaje que estamos proponiendo. Por ejemplo: ¿qué uso hacen los microemprendedores de las estrategias de comercialización? ¿Con cuáles problemas tienen que lidiar que no están enunciados en la teoría?, ¿qué grado de actualización tienen los contenidos enseñados en las aulas universitarias? ¿Qué problemas se presentan en las escuelas secundarias que las teorías del aprendizaje no resuelven? Consideramos que para aportar respuestas auténticas a estos interrogantes se requieren conocimientos disciplinares específicos junto con el conocimiento de la idiosincrasia de la comunidad de práctica.

Las PEEE son una oportunidad para que la formación universitaria establezca una relación dialéctica entre pensamiento y contexto y para que se desplace, aunque sea solo por un momento, de la encerrona cognitiva que se produce cuando los contenidos disciplinares se ofrecen solo en el espacio físico del aula. El proceso de construcción de conocimientos se amplifica y enriquece cuando ocurre mediante la participación de los sujetos en actividades cotidianas y en contextos reales. Las PEEE promueven una formación de puertas afuera relacionándose con una perspectiva del aprendizaje que incentiva a que el conocimiento se construya mediante la participación progresiva en la actividad y junto con otras personas. 


\section{Mitos sobre la transferencia y la aplicación del aprendizaje} El modo de pensamiento que se promueve en situaciones específicas introduce un marco teórico con nuevos referentes para reflexionar sobre la naturaleza del aprendizaje, del contexto, y el contenido de lo que se aprende. Las PEEE interpelan el arraigado concepto de transferencia de conocimientos y, por lo tanto, ponen en duda el supuesto de que lo que se aprende en las instituciones escolares se traslada y se aplica literalmente y sin mayores inconvenientes a otras situaciones y contextos distintos en los que fueron aprendidos.

La formación tradicional se sostiene en el mecanismo psicológico de la transferencia del aprendizaje. Esto explica la cantidad de teorías y herramientas metodológicas que se brinda a los estudiantes durante su formación con la firme convicción de que servirán en el futuro para analizar e intervenir en situaciones específicas. Este concepto de transferencia refleja ideas muy consolidadas sobre la continuidad entre entornos y sobre la uniformidad cultural. Se alega que la forma de intervenir y resolver problemas en las situaciones reales no es más que la aplicación de lo que se aprendió en el aula. El supuesto es que el conocimiento adquirido en circunstancias libres de influencias contextuales será fácilmente transportable y susceptible de desplegarse en cualquier otro contexto.

Ahora bien, si aceptamos la hipótesis de que la actividad adopta formas diferentes en situaciones específicas y que hay discontinuidad cognitiva entre contextos, tenemos motivos para suponer que lo que se aprende en el aula no se transfiere tan fácilmente a la vida real.

No estamos negando, ni siquiera poniendo en duda, la existencia de aprendizajes en las aulas. Estamos advirtiendo de las diferencias sustantivas entre el modo de aprender en el aula y el que tiene lugar en las actividades situadas en entornos reales. El respaldo empírico para esta advertencia proviene de las investigaciones recientes que exploran la cognición en la práctica. Los resultados de las investigaciones muestran que las mismas personas difieren en su modo de pensar cuando resuelven similares actividades pero en diferentes entornos. Esta afirmación nos lleva a pensar sobre el sentido de una formación universitaria tan alejado del contexto social y del contenido de la actividad del futuro profesional.

Quisiéramos insistir en la importancia de los contenidos disciplinares, de los problemas y temas en las PEEE. Dejarlos fuera del análisis sería desconocer su papel indiscutible como elemento clave del aprendizaje y del intercambio entre los sujetos en la práctica. El contenido es el punto de encuentro que posibilita la interacción entre los sujetos de la educación y los actores sociales.

Las PEEE no sustituyen hacer por aprender, en cada práctica hay algo que se enseña y algo que se aprende. Lo que se enseña y aprende no está capturado en una cápsula artificial, está en la comunidad de práctica. A través de las PEEE se aprenden los contenidos disciplinares alojados en problemáticas sociales, tienen un anclaje epistemológico además de un sentido social. Los contenidos adquieren una dimensión que conmueve el interés, el entusiasmo y el compromiso de los actores. Los estudiantes aprenden a interesarse por el valor de los contenidos a la vez que comprende sus sentidos.

Si bien es necesario que el o los profesores responsables de las prácticas planifiquen y prevean todo antes de su inicio, que especifiquen y regulen los comportamientos esperados, también es necesario tener una disposición a no querer saberlo todo de antemano. Esto permitirá trabajar creativamente con lo no previsto y abrir nuevos recorridos donde se resignifiquen teóricamente los hallazgos. Los profesores responsables de las prácticas tienen que ser capaces de aceptar que tanto los estudiantes como los actores sociales tienen un saber para aportar y un interés para participar, aunque no sepan por qué se interesarán y cómo las pondrán a trabajar. Estas imprecisiones son incómodas inicialmente pero alentadoras a la vez.

Las personas en situación interactúan con los contenidos de los problemas, esos contenidos están distribuidos entre los actores que de una u otra manera son partícipes del problema en cuestión. Pero no solo los contenidos están distribuidos sino también la cognición: la manera de pensarlos. En la comunidad de práctica hay una gran diversidad y heterogeneidad de formas de nombrar y resolver los problemas. La idea que los contenidos y las cogniciones están distribuidas tiene importantes efectos en la forma de concebir el aprendizaje.

Mientras que en los estudios tradicionales los procesos cognitivos son el resultado del esfuerzo intelectual individual para aprender contenidos abstractos y descontextualizados, la teoría de las cogniciones distribuidas propone otra matriz para pensar el aprendizaje: la progresiva participación en las comunidades de práctica y la apropiación de contenidos que resultan de la tensión teoría práctica. Esos contenidos están distribuidos en los programas de cada asignatura y en las prácticas de los actores de la comunidad.

El compromiso mutuo de los sujetos de la educación y los actores sociales en una práctica compartida se convierte paulatinamente y no sin inconvenientes, en contexto para el aprendizaje de los contenidos curriculares, y en oportunidad de modificar las creencias en conocimientos. La comunidad de práctica problematiza y transgrede lo esperable; interpela al conocimiento académico obligándolo a revisar sus propios argumentos, transformarlos o simplemente abandonarlos. Las PEEE son entonces un modo de aprender y, sobre todo, de crear nuevos conocimientos en el marco de cada disciplina y en la dimensión ética y política del ejercicio profesional. 


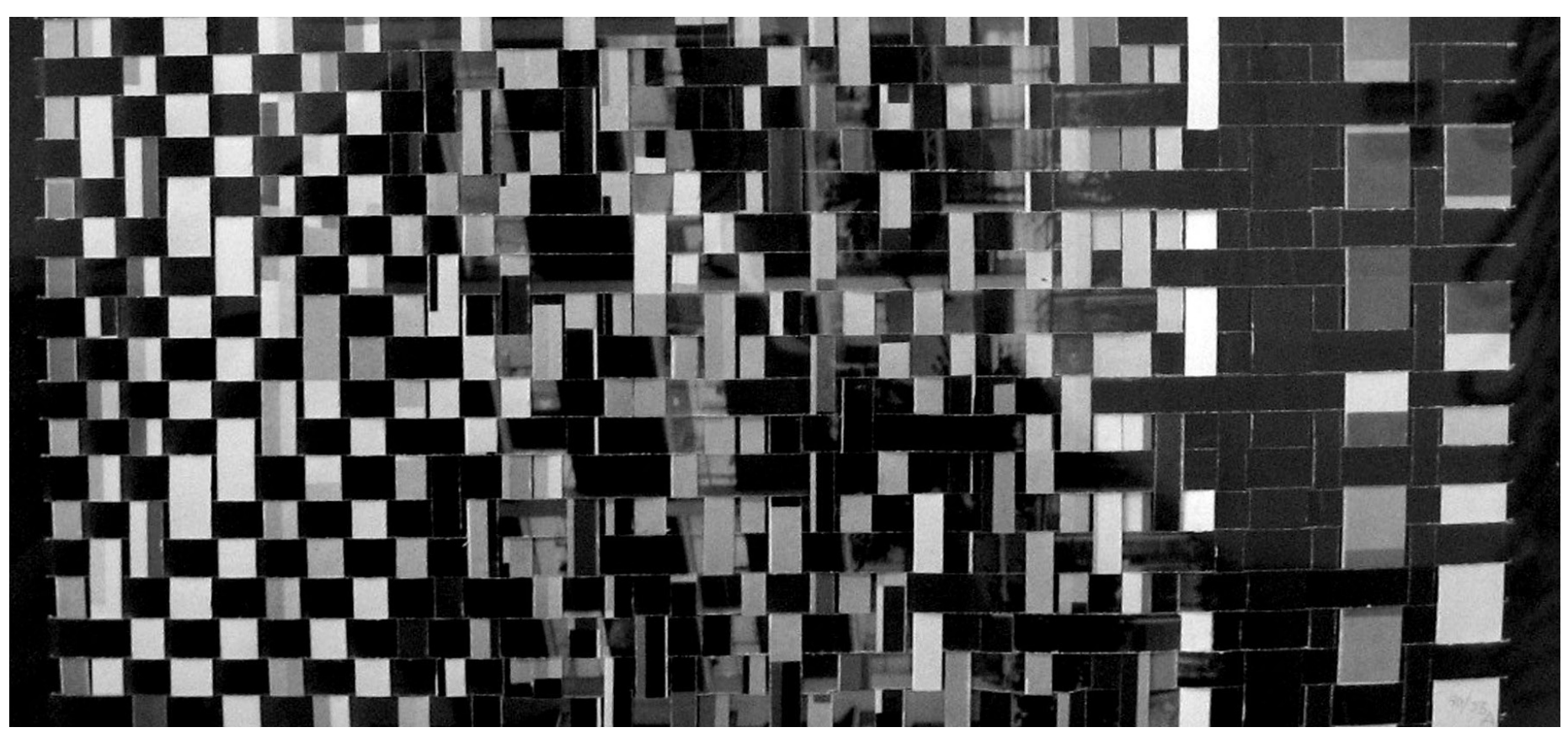

(C) Jorge Malachesky

6. La evaluación de los aprendizajes en las Prácticas de Extensión de Educación Experiencial

Las PEEE encuentran en la comunidad la heterogeneidad de lo cotidiano y en consecuencia el desafío de los sujetos de la educación es poner a trabajar las teorías y contenidos disciplinares en esa heterogeneidad. En las comunidades de prácticas el aprendizaje tiene distintas intensidades y se diversifica su trayectoria. A las negociaciones que dan inicio al trabajo en la comunidad de práctica las suceden el trabajo situado en la comunidad y a éste, el momento de reflexión teórica y subjetiva de la experiencia. No es el diseño ordenado ni la comunicación sin ruidos de contenidos abstractos la única propuesta que promueve la adquisición de saberes sino la acción, la discusión, la reflexión y la teorización como resultado de las ideas y venidas entre teoría y práctica; previstos e imprevistos, planificación y espontaneidad.

Estas ideas encuentran un límite en el formato de las evaluaciones de los aprendizajes convencionales actuales, por consiguiente, se impone como demanda la necesidad de pensar evaluaciones que integren los principios socio políticos y cognitivos que se promueven en PEEE.

Nos interesa describir y conceptualizar los componentes del acto evaluativo y formular algunos principios que deberían estar presentes en la construcción de propuestas evaluativas situadas y orientadas a evaluar los aprendizajes que se promueven a través de las PEEE.

Por ser una actividad de ocurrencia muy elevada en las instituciones educativas y más precisamente en los procesos de formación universitaria, la evaluación puede estudiarse de distintas maneras y enfocando distintos objetos, pero sin duda, todas coincidirán en que la evaluación tiene como función producir valoraciones. En el tema que nos ocupa, podemos sostener que la evaluación es una práctica intencionalmente planificada que tiene como propósito apreciar la calidad de los aprendizajes de los estudiantes. Para concretar el propósito se diseñan instrumentos que proporcionan información cuyo análisis e interpretación, mediado por referentes, conducen a la elaboración del juicio de valor.

En la afirmación anterior se mencionan los componentes centrales de la práctica evaluativa que nos interesa desplegar con mayor precisión:

- Para evaluar es necesario designar algo explícitamente como objeto: nombrar lo que se evaluará es convertirlo en objeto de evaluación: ¿qué se va a evaluar? La respuesta no es una afirmación sencilla, la precisión acerca de qué es el resultado de un proceso de discusión y reflexión y de diversas operaciones mediadas por distintas dimensiones de análisis. Por ejemplo, un primer grupo de interrogantes es: ¿se evaluará el conocimiento de los estudiantes del concepto y los tipos de estrategias de comercialización? ¿O el concepto y los modos de clasificar las teorías del aprendizaje? ¿O la aplicación que del concepto los estudiantes realizan? Otro interrogante será: ¿qué tiene mayor sentido: que los estudiantes aprendan conceptos o que aprendan a usarlos? Las respuestas a estas preguntas conducen al evaluador a la construcción de los referentes que serán punto de partida para emitir el juicio de valor.

- La precisión y publicidad de los referentes dan consistencia al acto evaluativo: los referentes son los principios desde los 


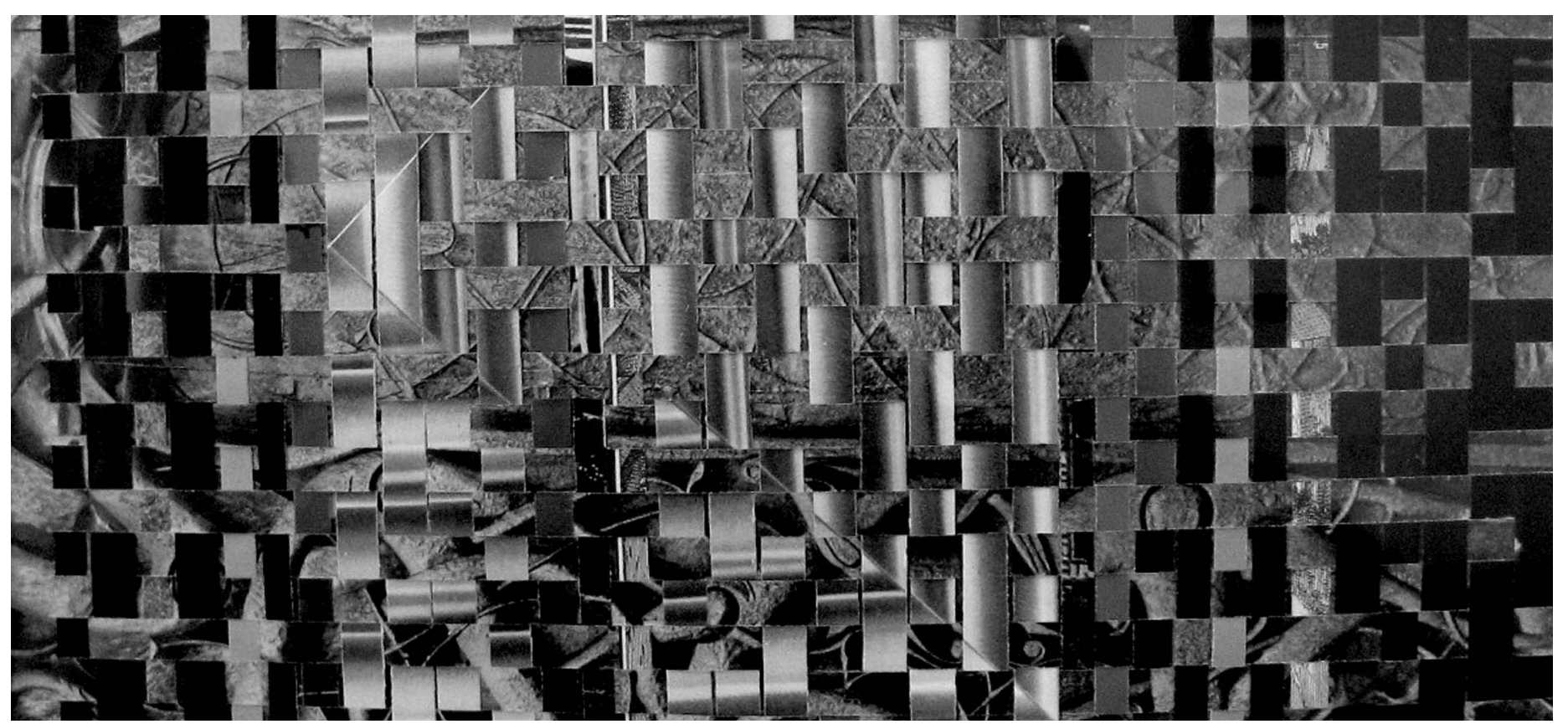

(C) Jorge Malachesky

cuales se produce o es susceptible de producirse la valoración. El evaluador debe asumir y comunicar, por ejemplo, de qué naturaleza es el aprendizaje que quiere que hagan los estudiantes universitarios. Luego, la valoración de la calidad del proceso de aprendizaje se realiza en tensión con el referente por el cual optó. La elección de los referentes es el resultado de un proceso específico de elaboración. La mayoría de las veces ese proceso no es explícito. La falta de claridad lleva a una gran inestabilidad del acto evaluativo marcado por la fragilidad de los juicios producidos. La evaluación necesita contar con información: la información no es independiente de los referentes. $Y$ es el evaluador quien genera la información que necesitará para apreciar la calidad del proceso y/o resultado de los aprendizajes de los estudiantes. En las evaluaciones convencionales el profesor es quien lleva a cabo el trabajo para que los estudiantes produzcan información. Las preguntas que se realizan en evaluación parcial, los problemas cuya solución se requiere en un examen final o las preguntas orales en clase, son ejemplos de cómo se genera información para la evaluación. El contenido de las respuestas de los estudiantes sirve a los profesores para conocer la calidad del proceso de aprendizaje y emitir un juicio de valor conforme a las escalas de calificación vigentes. Para los estudiantes es importante que la corrección de las evaluaciones proporcione explicaciones claras, lo cual permitirá entender su proceso y a la vez mejorarlo. - La evaluación acredita saberes: los profesores universitarios no pueden dejar de asumir esta responsabilidad. Podrán sí enriquecer los argumentos y explicar la variedad de la intensidad con la que se ponderan los aprendizajes pero no escapar a la calificación. Cuando son imprecisos los criterios con los que se juzga la producción de los estudiantes éstos se encuentran con apreciaciones tales como: "el proceso ha sido muy pobre" o "has realizado un muy buen trabajo", las que poco ayudan a entender el propio proceso de aprendizaje. La evaluación acredita saberes pero no debe cernirse únicamente a eso, hacerlo haría perder su sentido educativo. Cuando la evaluación es solo calificación se convierte en obsesión tanto para los profesores como para los estudiantes, la evaluación se burocratiza y se convierte en un acto más administrativo que educativo.

- La evaluación requiere una diversidad de instrumentos que sean sensibles a la naturaleza de los aprendizajes: los instrumentos de evaluación son los dispositivos que produce la información que luego de su análisis e interpretación permitirá la elaboración del juicio de valor. La situación de evaluación debiera ser una oportunidad real para que los alumnos puedan compartir lo que saben y aumentar las posibilidades de comprensión. En el apartado siguiente ampliamos este principio.

\section{Dar lugar a la innovación}

Históricamente, se clasificaron los instrumentos de evaluación en tradicionales y alternativos. La clasificación se hizo teniendo en cuenta la forma del instrumento, la naturaleza del conocimiento que se pretendía evaluar, el momento de aplicación y el propósito de la evaluación.

En el grupo de los tradicionales se incluyen los exámenes parciales y finales con preguntas cerradas. Pueden ser escritas $u$ orales, que pueden ser de opción múltiple, de V o F, de completar, etc. 
Por lo general son para resolver de manera individual y en clase, dependiendo la cantidad de preguntas del tiempo del que se disponga. En el de los alternativos, cualquier instrumento que salga de lo tradicional, por ejemplo: presentación de informes, escritura de diseños de proyectos, monografías, entre otros. En estos casos se da más libertad y autonomía a los estudiantes para que expongan sus saberes.

Consideramos que la opción por uno u otro tipo de instrumento debe realizarse una vez que se despeje la respuesta a la pregunta por el propósito y sentido de la evaluación. La elección de un tipo de instrumento por sobre otro o la elaboración de uno nuevo se realiza en relación con el propósito que se quiere que cumpla, con la naturaleza de la información que se quiere recabar y con el uso que se hará de esa información. Cualquiera de ellos, bien utilizado, cumple una función educativa importante. Las perversiones que se hayan hecho en el uso de los instrumentos, las injusticias en su elaboración y corrección o los sinsentidos en los que se haya caído, tienen relación con los caprichos del evaluador pero no con la validez del instrumento en sí.

En principio y a los efectos de avanzar con nuestra argumentación, podemos decir que el propósito de la evaluación es diseñar situaciones evaluativas integradas a las PEEE. Expusimos que en ellas el aprendizaje es el proceso y resultado de la apropiación progresiva de los saberes disciplinares que están distribuidos entre los sujetos de la educación y los actores sociales. La formación de los estudiantes ocurre mediante las intervenciones en situaciones reales; la observación, el diálogo, el debate, la reformulación de saberes adquiridos y/o la construcción de nuevas ideas. Ante tal diversidad de operaciones cognitivas la evaluación empobrecería el aprendizaje si se limita a solicitar reproducción de información. Es necesario que las evaluaciones incorporen preguntas que estimulen el pensamiento y que no lo limiten, hacer preguntas relevantes y significativas, esto es, indagar sobre lo que vale la pena seguir aprendiendo después del examen y, sobre lo que aún no se sabe. El mayor esfuerzo por parte del profesor será elaborar buenas preguntas, discernir cuál es el momento de formularlas y qué se espera que los estudiantes respondan.

\section{El relato de experiencia como instrumento de evaluación en la PEEE}

Definir con claridad lo que se quiere pedir a los estudiantes parece una tarea sencilla pero no lo es. Por un lado, está el compromiso ético de hacer explícitos los principios que orientan la práctica evaluativa; y por otra parte, porque redactar las consignas exigen un esfuerzo de escritura que muchas veces se desconoce. Las preguntas resultan obvias para quienes las redactan sin embargos sus destinatarios no las comprenden. Es así como el primer esfuerzo de los estudiantes en el momento de la evaluación es adivinar la intención de la pregunta del profesor, con el riesgo que eso acarrea.

En un mismo instrumento de evaluación se pueden incorporar preguntas de distintos grados de complejidad. Es necesario que las preguntas tengan información suficiente para que los estudiantes comprendan qué se les pide y entiendan el sentido de ello. Se valoran las preguntas abiertas por el grado de libertad que suponen sus respuestas; sin embargo, pueden resultar tramposas al momento de construir criterios justos de calificación. Si las consignas son muy amplias una opción es descomponerlas en partes, siempre que haya coherencia e integración entre ellas. El aprendizaje de los estudiantes que participan en PEEE podría evaluarse a través del relato de la experiencia. Consideramos que es un instrumento de particular importancia debido a que proporciona información sobre las dimensiones analíticas, reflexivas y comprensivas del proceso de conocimiento puesto en juego en la práctica experiencial. Es sensible para recuperar información de distintos momentos de la PEEE; desde datos descriptivos del contexto donde se desarrolló la práctica hasta el contenido de la reflexión que produce en los estudiantes la tensión teoría-práctica. Permite transformar la experiencia en conocimiento ordenado, fundamentado y comunicable.

El relato de la experiencia debe orientarse en torno a ejes o dimensiones que permitan presentar un recorte conceptual de los aprendizajes más significativos en relación con lo sucedido.

Es un instrumento que permite recuperar la voz e identidad de los actores sociales que fueron parte de la experiencia. Describir e interpretar lo que acontece en una comunidad de práctica ubica a los estudiantes en una posición comprometida con la situación. La escritura da cuenta de comprensión conceptual y también de afiliación social.

En tanto, este instrumento puede combinarse con otros o bien proponerse como único instrumento de evaluación que acompañe el proceso de adquisición de saberes durante la PEEE. En cualquiera de los casos, el profesor deberá proponer las preguntas que orienten la escritura de la experiencia y comunicar los criterios de valoración de los relatos que se producen.

Presentar el instrumento en esta página tiene el riesgo de perder la identidad que le otorga el trabajo situado en el proceso de la práctica y la autenticidad que le brinda la construcción colectiva con los colegas y los estudiantes. Es posible que la imagen no llegue a reflejar todo el movimiento que tuvo su construcción; no obstante, lo ofrecemos como ejemplo. 
PRÁCTICA DE EXTENSIÓN DE EDUCACIÓN EXPERIENCIAL: Relaciones que se construyen en las experiencias escolares. Condiciones socio-afectivas y cognitivas que las posibilitan.

CÁTEDRA: Psicología de la Educación. Facultad de Humanidades y Ciencias. UNL

\section{EVALUACIÓN FINAL}

Durante el desarrollo de la PEEE se realizaron distintas evaluaciones. Inicialmente, se seleccionaron casos extraídos de una investigación realizada en Argentina en la cual, a través de relatos autobiográficos, distintos jóvenes narraban sus experiencias de vida. Luego del análisis de los casos y de la interpretación de los mismos en relación con conceptos desarrollados en clase sobre adolescencia, juventud y vínculos, se publicaron las interpretaciones en el espacio de Foro que la cátedra tiene en el Campus virtual. Luego se les solicitó un informe escrito descriptivo y analítico sobre el resultado de la negociación inicial en la institución donde se llevaría a cabo la PEEE. Asimismo se realizó un parcial escrito individual, éste mantuvo el formato de análisis de caso, a la vez que indagó sobre concepciones teóricas desarrolladas en la cátedra. La evaluación final busca integrar las evaluaciones anteriores. En esta oportunidad se les solicita elaborar un escrito a través del cual deberán relatar la experiencia de la PEEE.

A continuación se ofrecen orientaciones para la escritura:

1. Describir las características de la PEEE propuesta por la cátedra; el escenario y los actores socials con los que interactuó.

2. a) Nombrar tres situaciones o problemas que hayan ocurrido en la PEEE y consideren relevantes analizar.

b) Explicar qué intervención tienen los actores sociales en las situaciones nombradas como problemas.

c) ¿Considera que los marcos teóricos desarrollados en la cátedra permiten analizar la situación? Explicar la opción que se adopte.

3. Realizar una interpretación de las representaciones iniciales sobre la PEEE, a la luz de la de la experiencia ya concluída.

4. Describir los aportes que hizo esta PEEE a su formación como estudiante universitario.

5. Mencione algunas sugerencias para mejorar o enriquecer esta experiencia.

\section{Conclusiones}

Las PEEE son una oportunidad para reflexionar sobre la naturaleza de la enseñanza el aprendizaje y la evaluación y, sobre la formación de profesionales. Ofrecen la oportunidad para que la formación universitaria salga de una "perspectiva teórica claustrofóbica" e interpelan a las concepciones convencionales sobre la formación universitaria.

En estas Prácticas comienza a visualizarse una nueva generación de problemas sobre los que la formación universitaria está dando poca o nula respuesta.

La inercia y la repetición sin variaciones de muchas prácticas universitarias están llevando a que los estudiantes se muestren apáticos, desinteresados o no comprometidos. En este sentido, las PEEE son un vínculo para reconstruir el entusiasmo por enseñar y aprender.

En relación con la evaluación, queremos insistir en que cambiar las formas de la evaluación es algo insuficiente si no cambian los principios que la sostienen. Para la elaboración de nuevos instrumentos de evaluación no hay recetas mágicas, se trata más bien de ir ensayando responsablemente formas alternativas que recuperen la experiencia y promuevan aprendizajes reflexivos.

\section{Aprender de la evaluación}

Debido al grado de novedad que los instrumentos de evaluación integrados a las PEEE tienen, es necesario disponer de un tiempo para evaluar su pertinencia y validez. O sea, para saber si el instrumento resultó legítimo para indagar sobe aquello que el profesor considera relevante y significativo preguntar. Es válida una evaluación cuando indaga sobre lo que se propone y cuando la información que aportan las respuestas de los estudiantes sirve para re orientar el aprendizaje y seguir enriqueciéndolo, cuando los estudiantes pueden dar cuenta del dominio que tienen de los conocimientos que adquirieron en la práctica experiencial y si son capaces de argumentar, reflexionar y discernir entre lo anecdótico y lo sustantivo.

\section{Referencias bibliográficas}

Chaiklin, L. y Lave, J. (Comps.) (2001). Estudiar las prácticas. Perspectivas sobre actividad y contexto. Buenos Aires: Amorrortu.

Engeström, Y. (2001). Los estudios evolutivos del trabajo como punto de referencia de la teoría de la actividad: el caso de la práctica médica de la asistencia básica. En Chaiklin, S. y Lave, J. (Comps.), Estudiar las prácticas. Perspectivas sobre la actividad y el contexto. Buenos Aires: Amorrortu

Lave, J. (1991). La cognición en la práctica. Barcelona: Paidós.

Salomon, G. (Comp.) (1993). Cogniciones distribuidas. Consideraciones psicológicas y educativas. Argentina: Amorrortu.

Wenger, E. (2001). Comunidades de práctica. Aprendizaje, significado e identidad. Madrid: Paidós 